\title{
Therapeutic Effects of Tocotrienol-Rich Fraction on Rhinitis Symptoms in Platelet-Activating Factor Induced Allergic Rhinitis
}

Cheryl Wei Ling Teo ( $\square$ weiling.teo@davoslife.com )

Davos Life Science https://orcid.org/0000-0003-4458-6376

\section{Stephanie Jia Ying Png}

Nanyang Technological University

Yee Wei Ung

KLK: Kuala Lumpur Kepong Berhad

Wei Ney Yap

Davos Life Science

\section{Research Article}

Keywords: Allergic rhinitis, Tocotrienol-rich fraction, Tocotrienol, Antioxidant, Anti-inflammation, Antiallergic, Platelet-activating factor

Posted Date: February 1st, 2022

DOI: https://doi.org/10.21203/rs.3.rs-1258949/v1

License: (9) This work is licensed under a Creative Commons Attribution 4.0 International License. Read Full License 


\section{Abstract}

Background: Platelet-activating factor (PAF) has been suggested to be a potent inflammatory mediator in Allergic rhinitis (AR) pathogenesis. Vitamin $\mathrm{E}$ is known as a potential therapeutic agent for airway allergic inflammation. This study aimed to investigate the beneficial effects of palm-derived Tocotrienol-rich fraction (TRF) on PAF-induced AR in a rat model.

Methods: Sprague Dawley rats were randomly assigned into 3 groups: Control, PAF-induced AR and PAFinduced AR with TRF treatment. To induce AR, $50 \mu \mathrm{l}$ of $16 \mu \mathrm{g} / \mathrm{ml}$ PAF was nasally instilled into each nostril. From day 1 to 7 after AR induction, $10 \mu$ of $16 \mu \mathrm{g} / \mu \mathrm{l}$ TRF was delivered intranasally to the TRF treatment group. Complete upper skulls were collected for histopathological evaluation on day 8.

Results: The average severity scores of AR were significantly higher in the PAF-induced AR rats compared to both control and PAF-induced AR with TRF treatment. The histologic examination of the nasal structures showed moderate degree of inflammation and polymorphonuclear cells infiltration in the lamina propria, mucosa damage and vascular congestion in the PAF-induced AR rats. TRF was able to ameliorate the AR symptoms by restoring the nasal structures back to normal. H\&E staining demonstrated a statistically significant benefit upon TRF treatment, where minimal degree of inflammation, and a reduction in the infiltration of polymorphonuclear cells, mucosa damage and vascular congestion were observed.

Conclusion: TRF exhibited symptomatic relief action in AR potentially due to its antioxidant, antiinflammatory and anti-allergic properties.

\section{Introduction}

Allergic rhinitis (AR) is a common inflammatory disorder of the nasal mucosa that is clinically symptomized by sneezing, nasal itching, rhinorrhoea, and nasal congestion (1). It has been suggested as a prevalent yet undertreated respiratory disease affecting up to $30 \%$ of adults and $40 \%$ of children over the world (2). AR substantially impacts the socioeconomic burden in terms of increased healthcare costs and decreased productivity, as well as impairment in quality of life, causing public health concerns $(3,4)$.

The pathophysiology of AR involves a complex Immunoglobulin E (IgE)-mediated hypersensitivity reaction triggered by inhaled allergens. Upon exposure, immune cells process the allergens and stimulate B-cell mediated immune response. The cross-linking of IgE antibody on the surface of the activated inflammatory cells trigger mast cell degranulation in the early phase of allergic response, releasing preformed and newly synthesized mediators such as platelet-activating factor (PAF), histamine and proinflammatory cytokines that promote vascular permeability, rhinorrhoea and vasodilation $(5,6)$. These mediators perpetuate the allergic response to the late phase, triggering cellular inflammation. The recruitment of several inflammatory cells, for instance the eosinophils, basophils, monocytes and lymphocytes, continues promoting the release of the mediators and remodel of the nasal tissues. This resulted in nasal congestion, the most common symptom reported by AR patients (7). PAF is a potent 
lipid inflammatory mediator released by several cell types (8), whose role was first reported in literature by a French immunologist, Jacques Benveniste, as a mediator of anaphylaxis (9). In AR, PAF has been recognized to have an important role in inducing vascular permeability associated with rhinorrhoea and nasal congestion, consequently the nasal hyperreactivity is then encouraged $(10,11)$. Furthermore, PAF serves as a powerful chemoattractant for eosinophils and neutrophils to the site of allergic inflammation. PAF induces leukocytes degranulation and adhesion, triggering the production and release of free radicals such as superoxide anion and hydroxyl anions in the nasal mucosa (12). Overproduction of the free radicals generates oxidative stress (OS) and exacerbates inflammation in the cell. As such, therapeutic antioxidants could potentially be adopted to provide better control of the inflammation in AR and prevent symptoms aggravation.

Vitamin $E$ is a robust lipid soluble antioxidant that presents peroxyl radical-scavenging activity (13). It comprises of 2 homologs, Tocopherol (TP) and Tocotrienol (T3), with each homolog further classified into $a, \beta, y$ and $\delta$ isoforms. TP and T3 have the same basic chemical structure characterized by a long chain hydrocarbon at the 2-position of a chromanol ring. TP consists of a side chain made of a phytylin group while T3 consists of an isoprenyl group with 3 unsaturated double bonds at positions $3^{\prime}, 7^{\prime}$ and $11^{\prime}$ $(14,15)$. Both TP and T3 display antioxidant properties, where T3 has shown a more potent free radical scavenging effect than TP due to their better penetration and distribution in the lipid layers of the cell membranes (16-18). Begum et al. concluded a higher incorporating activity of a-T3 than a-TP resulting in an increased protection against erythrocyte oxidation and impairing its deformability (19). Furthermore, Tocotrienol-rich fraction (TRF) at $50 \mu \mathrm{M}$ was reported to show a greater protective effect against LDL oxidation than TP in a cell line study (20). Evidences also indicated T3 to be more superior than TP in anti-inflammation by lowering the proinflammatory mediators and suppressing the proinflammatory signaling such as nuclear factor kappa B (NF-KB) and signal transducer and activator of transcription 3 (STAT3) (21-23).

Currently, therapeutic interventions of AR include pharmacotherapy and immunotherapy (24). According to the Allergic Rhinitis and Its Impact on Asthma (ARIA) guidelines, intranasal corticosteroids are the first line medication for mild to moderate AR. In more serious cases, second line medications such as oral antihistamines and nasal decongestant or allergen immunotherapy could be considered (25). However, there are undesired side effects with long term use of these AR treatments. Vitamin $E$ has been known as a potential therapeutic agent for airway allergic inflammation, though TP was dominantly focused in most of the research (26-28). This study investigated the influence of PAF on the development of AR in an animal model. Subsequently, the efficiency of intranasal administration of palm-derived TRF in alleviating the symptoms of AR and histological changes in the nasal structures were determined. By elucidating the pharmacotherapy benefits of TRF, this study may help to develop TRF as a therapeutic agent in the AR management.

\section{Methods}

\section{Drugs and chemicals}


Tocotrienol-rich fraction (TRF) was obtained from Davos Life Science Sdn. Bhd. (Malaysia). Plateletactivating factor (PAF), bovine serum albumin (BSA), glycerine, tween-80, ketamine chloride, xylazine hydrochloride and pentobarbital were obtained from Sigma-Aldrich (St. Louis, MO, USA).

\section{Animal model}

This study was conducted with male Sprague Dawley rats weighing between 250 to $300 \mathrm{~g}$, that were without any evidence of upper airway infection. The rats were housed in the Biological Resource Centre (BRC), $A^{\star}$ Star Singapore under standard laboratory conditions $\left(12 \mathrm{~h} \mathrm{light} / 12 \mathrm{~h}\right.$ dark schedule at $24^{\circ} \mathrm{C} \pm$ $\left.2^{\circ} \mathrm{C}\right)$. The rats were fed with standard pellet diet and water ad libitum. This study was approved by the BRC Institutional Animal Care and Use Committee (IACUC).

\section{Allergic rhinitis (AR) model}

The rats were acclimatized to laboratory conditions for $72 \mathrm{~h}$ prior to experiment and randomly assigned to 3 groups. Group 1 is the control group where the healthy rats were not exposed to the AR. Group 2 represents the PAF-induced AR group where the rats were intranasally induced with $50 \mu \mathrm{l}$ of $16 \mu \mathrm{g} / \mathrm{ml}$ PAF per nostril. Group 3 is the PAF-induced AR with TRF treatment group where the AR rats additionally received $10 \mu \mathrm{l}$ of $16 \mu \mathrm{g} / \mu \mathrm{l}$ TRF per nostril.

The experimental design for the AR induction and treatment was shown in Figure 1. Briefly, the rats were subjected to a combination of $75 \mathrm{mg} / \mathrm{kg}$ of ketamine chloride and $10 \mathrm{mg} / \mathrm{kg}$ of xylazine hydrochloride anaesthesia intraperitoneally. Isoflurane inhalational anaesthetic agent was avoided to prevent irritation of the nasal mucosa. AR was induced via a one-time induction of PAF to the nasal cavity. The PAF stock was prepared in absolute ethanol and subsequently diluted with phosphate-buffered saline containing $0.25 \%$ BSA to working concentration. On day $0,50 \mu \mathrm{l}$ of $16 \mu \mathrm{g} / \mathrm{ml}$ PAF was instilled into each nostril of the AR-induced rats by placement of small droplets using a micropipette. The same amount of vehicle was applied to the control group.

From day 1 to 7 post-induction, the AR-induced rats were administered with $10 \mu$ of excipient solution containing $5 \%$ saline, $5 \%$ glycerine, $5 \%$ tween- 80 and $85 \%$ water into each nostril. While the TRF treatment group received additional $16 \mu \mathrm{g} / \mu \mathrm{l}$ of TRF in the same excipient solution at $10 \mu \mathrm{l}$ per nostril. No treatment was given to the control group.

\section{Histology examination}

All the rats were euthanized with $120 \mathrm{mg} / \mathrm{kg}$ of pentobarbital on day 8 . The rats were decapitated and the eyes, skin, fur, muscle tissues, and the mandibles were excised. The complete upper skull was then fixed in $10 \%$ buffered formalin. The samples were dehydrated in ascending grades of alcohol and xylene and embedded in paraffin for histological examination by Advanced Molecular Pathology Laboratory (AMPL), $A \star S T A R$. Tissue sections were taken from paraffin blocks at $5 \mu \mathrm{m}$ thickness and stained with hematoxylin and eosin (H\&E). The stained samples were evaluated with a light microscope by a certified veterinary pathologist blinded to the protocol. 


\section{Scoring of AR severity}

The significance of nonneoplastic lesions in nasal tissues was evaluated semi quantitatively by applying grading scheme of 0 to 5 severity grades with reference to previous reports $(29,30)$. Severity grading is considered to be semi quantitative as it relies on the estimation of severity rather than actual measurement.

\section{Statistical analysis}

The data were analysed using Graphpad Prism version 9.1.0 (Graphpad Softare, San Diego, CA). Comparison of the histological evaluation graded by the veterinary pathologist in all groups were performed with one-way ANOVA and subsequently post-hoc Tukey test. Statistical significance value was set at $p<0.05$.

\section{Results}

\section{TRF reduced average severity scores of AR in PAF-induced rats}

Figure 2 shows the average severity scores of AR in the Control, PAF-induced AR and PAF-induced AR with TRF treatment group. Overall, PAF aggravated the AR symptoms by showing the highest average severity score of 0.56 , while TRF was able to mitigate the damage significantly by lowering the severity score to 0.27 that is close to control $(0.26)$.

\section{TRF showed protective effects against AR symptoms in PAF-induced rats}

The histological evaluation of the nasal cavity, nasal septum and nasal turbinate including degree of inflammation, inflammatory cells infiltrate and mucosa damage in all groups are presented in Figure 3. The severity of vascular congestion in submucosa is shown in Figure 4.

\section{Control group}

In the control group, none to minimal degree of inflammation, polymorphonuclear cells infiltration and mucosa damage were observed (Figure 3A). H\&E staining demonstrated intact epithelial and regular goblet cells in the nasal septum and turbinate (Figure 3B). Results for severity score (Figure 4A) and H\&E staining (Figure 4B) showed the presence of mild vascular congestion in the submucosa. This is most likely a background change within normal limits.

\section{PAF-induced AR group}


Compared to the control group, there was a greater degree of inflammation and significant submucosal infiltration of polymorphonuclear cells in the PAF-induced AR group, with $p<0.05$. An extensive damage in the mucosa was significantly observed as compared to the control and TRF treated group, with $p<0.0001$ (Figure 3A). H\&E staining demonstrated aggregates of polymorphonuclear cells as well as the deprivation of epithelial and goblet cells due to substantial mucosa damage (Figure 3C). Moreover, vascular congestion was significantly exacerbated by the PAF in AR rats (Figure $4 \mathrm{~A}$ and $4 \mathrm{C}$ ).

\section{PAF-induced AR group with TRF treatment}

The overall histological parameters in the PAF-induced AR group receiving TRF treatment were restored to the regular nasal structures close to the healthy control, particularly in mucosa damage with $p<0.0001$ (Figure 3A). There was a marked improvement in the histological changes demonstrated by the H\&E staining. A lower degree of inflammation, minimal infiltration of polymorphonuclear cells and minimal degree of mucosa damage were observed in the nasal structure (Figure 3D). Furthermore, TRF significantly improved the vascular congestion when compared to PAF-induced AR group, with $p<0.05$ (Figure 4A and 4D).

\section{Discussion}

Oxidative stress (OS) and inflammation are intricately linked where OS is intensified upon triggering inflammation (31). The imbalance of antioxidants and free radicals such as reactive oxygen species (ROS) has been suggested to play a vital role in the pathogenesis of allergic diseases, for example asthma, wheezing and atopic dermatitis, but little focus was given to Allergic rhinitis (AR) (32-35). With the concept of 'one airway, one disease', it is likely that OS and inflammation take part in AR considering the upper and lower airways often share common disease associations and immunopathological mechanisms (36).

Current evidence suggests the involvement of PAF in the generation of ROS and acts as a powerful stimulator of acute inflammatory processes in allergic reactions $(12,37)$. A rat model of PAF-induced rhinosinusitis has been successfully established by Jeon et al. where the presence of neutrophil clusters in nasal cavity, infiltration of polymorphonuclear cells in mucosa, goblet cell hyperplasia in the epithelium and epithelial damage were observed to be peak at day 3 after one-time intranasal administration of 16 $\mu \mathrm{g} / \mathrm{ml}$ PAF at $50 \mu \mathrm{l}$ (38). Besides, several human studies involving AR patients have proved the potency of PAF in inducing rhinitis like symptoms including rhinorrhoea and nasal congestion, as well as increasing eosinophilic and neutrophilic infiltration. PAF was also found in AR patients' nasal secretions after antigen challenge of upper respiratory airway (39). Moreover, the involvement of PAF in inducing oxidative bursts, priming polymorphonuclear cells for production and releasing of ROS was demonstrated $(40,41)$. In line with these, Kato et al. reported a significant cellular adhesion and $\mathrm{O}_{2}$ - release in PAFinduced human eosinophils (42). All things considered, the engagement of PAF in allergic inflammation of airways in humans is plausible. In the current study, the PAF-induced AR model has been adopted to elucidate the role of PAF in the pathogenesis of AR. PAF has exacerbated AR symptoms in the AR group, 
especially in the infiltration of polymorphonuclear cells, mucosa damage and vascular congestion. Although ovalbumin (OVA) is one of the most widely used inducers for establishment of allergic respiratory in animal models, it acts as a multisystemic inducer and is administered in the presence of an adjuvant, with symptoms peaking after a period of time (43). Unlike OVA, PAF induced nasal challenge can be done in a single dose with the appearance of the symptoms locally (44).

In the present study, we demonstrated the protective actions of TRF in ameliorating AR symptoms in a PAF-induced AR rat model which, to the best of our knowledge, is the first time in literature. The dosage of TRF in the range of 5 to $16 \mu \mathrm{g} / \mu \mathrm{l}$ delivered at $10 \mu \mathrm{l}$ was examined, with reference to the gold standard treatment of using nasal corticosteroids. Mometasone furoate (MF) as an intranasal aqueous spray is among the most common prescribed corticosteroids for AR. Its efficacy has been evaluated in several randomized, double-blind, placebo-controlled clinical trials involving adults and children with AR. Overall clinical data show MF aqueous spray, administered once daily at $100 \mu \mathrm{g}$ per nostril for 2-12 weeks, is not only effective in treating AR, but also prevents the onset of symptoms in AR patients $(45,46)$. In our work, TRF at highest dose of $160 \mu \mathrm{g}$ markedly restored the nasal structures of the PAF-induced AR rats to normal as the healthy rats. Results from this study suggested TRF, as a natural phytonutrient, may provide symptomatic reliefs that is comparable to MF in the management of AR.

T3 possesses both antioxidant and anti-inflammatory properties. It not only can neutralize free radicals, but also has an anti-inflammatory action in allergic disorders $(47,48)$. In a house dust-mite asthmainduced mouse model, Peh et al. demonstrated $250 \mathrm{mg} / \mathrm{kg}$ body weight of palm extracted $\mathrm{\gamma}$-T3 given via oral gavage acts as a direct free radical scavenger by preventing airway inflammation and OS effectively as compared to $\mathrm{a}$-TP. In addition, $\mathrm{Y}$-T3 was able to inhibit the NF-KB nuclear translocation and promoted the levels of nuclear factor erythroid 2-related factor 2 (Nrf2). The results indicated the therapeutic potential of T3 for the treatment of allergic diseases (49). In an OVA challenged allergic asthma model using Brown Norway rats, Zainal et al. reported oral supplementation of palm-derived TRF at $30 \mathrm{mg} / \mathrm{kg}$ body weight modulated the inflammatory reaction in bronchial asthma through the inhibition of Th2derived proinflammatory cytokines production (50). In another study, Wagner et al. described the therapeutic effects of dietary $Y$-TP supplementation in a rodent model of ozone-enhanced allergic nasal responses. Rats treated with oral $\mathrm{Y}$-TP at $100 \mathrm{mg} / \mathrm{kg}$ body weight showed a decrease in the intraepithelial mucosubstances, eosinophilic inflammation and mucus-stimulating factors such as cysteinyl leukotrienes. This findings suggested the ability of TP in attenuating the effects of ozone in allergic upper airways of AR rat models (51). Albeit TP was employed as the treatment agent in Wagner's study, the hypothesis that T3, which possess 40-60 times stronger antioxidant and anti-inflammatory properties than TP (52), could provide protection against AR via similar mechanisms is plausible.

At the moment, there is a lack of intervention human trial into the management of AR using T3. Nevertheless, the relationship between dietary vitamin E and allergic diseases in humans has been previously reported. Vitamin E supplementation was shown to protect young children from the development of atopy and wheezing (53). In a recent cross-sectional study, 65 children with AR and 48 healthy controls aged 6-14 years were assessed for the association between serum level of vitamin $E$ and 
the occurrence and severity of AR (54). The results showed a significantly lower serum vitamin E levels in children with AR than in the normal children, and a negative correlation was found between level of vitamin $\mathrm{E}$ in serum and specific IgE along with skin prick test grade. Several studies have also reported a decrement of vitamin $\mathrm{E}$ and vitamin $\mathrm{C}$ level and an increment of the oxidized glutathione in the airway lining fluids of allergic airway diseases models $(55,56)$.

More molecular studies could have been done to better comprehend the mode of action of TRF against AR. Firstly, the proinflammatory cytokines related to AR could be evaluated. Secondly, the assessment of signalling pathways, for instance the Phosphatidylinositol 3-kinase/protein kinase B (PI3K/Akt), would be helpful to validate the potential mechanism of antioxidant and anti-allergic function of T3. Lastly, a randomized controlled human clinical trial investigating the evidence acquired from this experimental animal study may provide a new option in AR management.

\section{Conclusion}

TRF exhibited symptomatic relief actions in AR potentially due to its antioxidant, anti-inflammatory and anti-allergic properties. This is the first study to demonstrate the significance of intranasally administered TRF which may offer an alternative therapeutic option in the prevention and reversal of AR.

\section{Abbreviations}

a-T3: Alpha-tocotrienol; a-TP: Alpha-tocopherol; $y$-T3: Gamma-tocotrienol; ANOVA: Analysis of variance; AR: Allergic rhinitis; ARIA: Allergic rhinitis and its impact on asthma; BSA: Bovine serum albumin; H\&E: Hematoxylin and eosin; IgE: Immunoglobulin E; MF: Mometasone furoate; NF-кB: Nuclear factor kappa-light-chain-enhancer of activated B cells; OS: Oxidative stress; OVA: Ovalbumin; PAF: Platelet-activating factor; PI3K/Akt: Phosphatidylinositol 3-kinase/protein kinase B; ROS: Reactive oxygen species; STAT 3: Signal transducer and activator of transcription 3; T3: Tocotrienol; TP: Tocopherol; TRF: Tocotrienol-rich fraction

\section{Declarations}

\section{Ethics approval and consent to participate}

All animal experiments were approved by the Biological Resource Centre (BRC) Institutional Animal Care and Use Committee (IACUC) under protocol \#191422.

\section{Consent for publication}

Not applicable.

\section{Availability of data and materials}


The data analysed and used in this study are available from the corresponding authors upon reasonable request.

\section{Competing interests}

Cheryl Wei Ling Teo and Wei Ney Yap work for Davos Life Science (a subsidiary of KLK Oleo). Yee Wei Ung works for KLK Oleo, a manufacturer of tocotrienols. Stephanie Jia Ying Png has no conflict of interest to declare.

\section{Funding}

This work was financially supported by a research grant from KL-Kepong Oleomas Sdn. Bhd. (KLK Oleo) to Davos Life Science.

\section{Author's contributions}

Cheryl Wei Ling Teo and Wei Ney Yap participated in the design of the study, experiment execution and data analysis. Cheryl Wei Ling Teo and Stephanie Jia Ying Png were responsible for drafting the manuscript. Yee Wei Ung critically revised the work for important intellectual content. All authors provided final approval of the version to be published and agree to be accountable for all aspects of the work.

\section{Acknowledgements}

We would like to thank the staffs from the Biological Resource Centre (BRC), $A^{\star}$ Star and Histopathology/Advanced Molecular Pathology Lab (AMPL) for their scientific insight and technical support.

\section{References}

1. Bousquet J, Anto JM, Bachert C, Baiardini I, Bosnic-Anticevich S, Walter Canonica G, et al. Allergic rhinitis. Nat Rev Dis Prim. 2020 Dec;3(1):95. 6(.

2. Passali D, Cingi C, Staffa P, Passali F, Muluk NB, Bellussi ML. The International Study of the Allergic Rhinitis Survey: outcomes from 4 geographical regions. Asia Pac Allergy. 2018 Jan 25;8(1):e7.

3. Dierick BJH, van der Molen T, Flokstra-de Blok BMJ, Muraro A, Postma MJ, Kocks JWH, et al. Burden and socioeconomics of asthma, allergic rhinitis, atopic dermatitis and food allergy. Expert Rev of Pharmacoecon Outcomes Res. 2020 Oct;20(5):437-53.

4. Kulthanan K, Chusakul S, Recto MT, Gabriel MT, Aw DCW, Prepageran N, et al. Economic burden of the inadequate management of allergic rhinitis and urticaria in Asian countries based on the GA2LEN model. Allergy Asthma Immunol Res. 2018 Jul;10(4):370-8.

5. Min YG. The pathophysiology, diagnosis and treatment of allergic rhinitis. Allergy Asthma Immunol Res. 2010 Apr;2(2):65-76. 
6. Sin B, Togias A. Pathophysiology of allergic and nonallergic rhinitis. Proc Am Thorac Soc. 2011 Mar;8(1):106-14.

7. Small P, Keith PK, Kim H. Allergic rhinitis. Allergy Asthma Clin Immunol. 2018 Sep; 12;14 (Suppl 2):51.

8. Braquet $P$, Rola-Pleszcynski M. Platelet-activating factor and cellular immune responses. Immunol Today. 1987;8(11):345-51.

9. Pawankar R, Mori S, Ozu C, Kimura S. Overview on the pathomechanisms of allergic rhinitis. Asia Pac Allergy. 2011 Oct;1(3):157-67.

10. Alfaro V. Role of histamine and platelet-activating factor in allergic rhinitis. J Physiol Biochem. 2004 Jun;60(2):101-11.

11. Muñoz-Cano R, Valero A, Roca-Ferrer J, Bartra J, Sanchez-Lopez J, Mullol J, et al. Platelet-activating factor nasal challenge induces nasal congestion and reduces nasal volume in both healthy volunteers and allergic rhinitis patients. Am J Rhinol Allergy. 2013 Mar-Apr;27(2):e48-52.

12. Muñoz-Cano RM, Casas-Saucedo R, Valero Santiago A, Bobolea I, Ribó P, Mullol J. Platelet-Activating Factor (PAF) in Allergic Rhinitis: Clinical and Therapeutic Implications. J Clin Med. 2019 Aug;29(9):1338. 8(.

13. Rizvi S, Raza ST, Ahmed F, Ahmad A, Abbas S, Mahdi F. The role of Vitamin E in human health and some diseases. Sultan Qaboos Univ Med J. 2014 May;14(2):e157-65.

14. Xu Z, Harvey KA, Pavlina TM, Zaloga GP, Siddiqui RA. Tocopherol and tocotrienol homologs in parenteral lipid emulsions. Eur J Lipid Sci Technol. 2015 Jan;117(1):15-22.

15. Shahidi F, De Camargo AC. Tocopherols and tocotrienols in common and emerging dietary sources: Occurrence, applications, and health benefits. Int J Mol Sci. 2016 Oct 20;17(10):1745.

16. Serbinova EA, Packer L. Antioxidant properties of a-tocopherol and a-tocotrienol. Methods Enzymol. 1994;234:354-66.

17. Suzuki YJ, Tsuchiya M, Wassall SR, Choo YM, Govil G, Kagan VE, et al. Structural and dynamic membrane properties of alpha-tocopherol and alpha-tocotrienol: implication to the molecular mechanism of their antioxidant potency. Biochemistry. 1993 Oct 12;32(40):10692-9.

18. Sen CK, Khanna S, Roy S. Tocotrienols. Vitamin E beyond tocopherols. Life Sci. 2006 Mar 27;78(18):2088-98.

19. Begum N, Terao J. Protective effect of a-tocotrienol against free radical-induced impairment of erythrocyte deformability. Biosci Biotechnol Biochem. 2002 Feb;66(2):398-403.

20. Mutalib MSA, Khaza'ai $H$, Wahle KWJ. Palm-tocotrienol rich fraction (TRF) is a more effective inhibitor of LDL oxidation and endothelial cell lipid peroxidation than a-tocopherol in vitro. Food Res Int. 2003;36(5):405-13.

21. Ahsan $H$, Ahad A, Iqbal J, Siddiqui WA. Pharmacological potential of tocotrienols: a review. Nutr Metab (Lond). 2014 Nov;12(1):52. 11(.

22. Ng LT, Ko HJ. Comparative effects of tocotrienol-rich fraction, a-tocopherol and a-tocopheryl acetate on inflammatory mediators and nuclear factor kappa B expression in mouse peritoneal 
macrophages. Food Chem. 2012;Sep15(2):920-5. 134(.

23. Wu S-J, Liu P-L, Ng L-T. Tocotrienol-rich fraction of palm oil exhibits anti-inflammatory property by suppressing the expression of inflammatory mediators in human monocytic cells. Mol Nutr Food Res. 2008 Aug;52(8):921-9.

24. Bernstein DI, Schwartz G, Bernstein JA. Allergic Rhinitis: Mechanisms and Treatment. Immunol Allergy Clin North Am. 2016 May;36(2):261-78.

25. Bousquet $J$, Van Cauwenberge P. Allergic Rhinitis and its Impact on Asthma (ARIA) in collaboration with the World Health Organisation. Prim Care Respir J. 2002 Mar;11(1):18-9.

26. Geiser M, Lay JC, Bennett WD, Zhou H, Wang X, Peden DB, et al. Effects of ex vivo y-tocopherol on airway macrophage function in healthy and mild allergic asthmatics. $J$ Innate Immun. 2013;5(6):613-24.

27. Burbank AJ, Duran CG, Pan Y, Burns P, Jones S, Jiang Q, et al. Gamma tocopherol-enriched supplement reduces sputum eosinophilia and endotoxin-induced sputum neutrophilia in volunteers with asthma. J Allergy Clin Immunol. 2018 Apr;141(4):1231-8.e1.

28. Wagner JG, Jiang Q, Harkema JR, Illek B, Patel DD, Ames BN, et al. Ozone enhancement of lower airway allergic inflammation is prevented by $\mathrm{Y}$-tocopherol. Free Radic Biol Med. 2007 Oct;15(8):1176-88. 43(.

29. Shackelford C, Long G, Wolf J, Okerberg C, Herbert R. Qualitative and quantitative analysis of nonneoplastic lesions in toxicology studies. Toxicol Pathol. 2002 Jan-Feb;30(1):93-6.

30. Klopfleisch R. Multiparametric and semiquantitative scoring systems for the evaluation of mouse model histopathology - a systematic review. BMC Vet Res. 2013 Jun;21:9:123.

31. Lugrin J, Rosenblatt-Velin N, Parapanov R, Liaudet L. The role of oxidative stress during inflammatory processes. Biol Chem. 2014 Feb;395(2):203-30.

32. Celik M, Tuncer A, Soyer OU, Saçkesen C, Tanju Besler H, Kalayci O. Oxidative stress in the airways of children with asthma and allergic rhinitis. Pediatr Allergy Immunol. 2012 Sep;23(6):556-61.

33. Kompauer I, Heinrich J, Wolfram G, Linseisen J. Association of carotenoids, tocopherols and vitamin $\mathrm{C}$ in plasma with allergic rhinitis and allergic sensitisation in adults. Public Health Nutr. 2006 Jun;9(4):472-9.

34. Devereux G, Seaton A. Diet as a risk factor for atopy and asthma. J Allergy Clin Immunol. 2005 Jun;115(6):1109-17.

35. Jaffary F, Faghihi G, Mokhtarian A, Hosseini SM. Effects of oral vitamin E on treatment of atopic dermatitis: A randomized controlled trial. J Res Med Sci. 2015 Nov;20(11):1053-7.

36. Grossman J. One airway, one disease. Chest. 1997 Feb;111(2 Suppl):11S-16S.

37. Kurose I, Argenbright LW, Wolf R, Granger DN. Oxidative Stress during Platelet-Activating FactorInduced Microvascular Dysfunction. Microcirculation. 1996 Dec;3(4):401-10.

38. Jeon S-Y, Kim J-P, Ahn S-K, Kim E-A, Kim B-G. Rat Model of Platelet-Activating Factor-Induced Rhinosinusitis. Ann Otol Rhinol Laryngol. 2005 May;114(5):393-8. 
39. Miadonna A, Tedeschi A, Arnoux B, Sala A, Zanussi C, Benveniste J. Evidence of PAF-acether metabolic pathway activation in antigen challenge of upper respiratory airways. Am Rev Respir Dis. 1989 Jul;140(1):142-7.

40. Takahashi S, Yoshikawa T, Naito Y, Tanigawa T, Yoshida N, Kondo M. Role of Platelet-Activating Factor (PAF) in Superoxide Production by Human Polymorphonuclear Leukocytes. Lipids. 1991 Dec;26(12):1227-30.

41. Miike S, Kurasawa K, Saito Y, Iwamoto I. Platelet-activating factor activates mitogen-activated protein kinases through the activation of phosphatidylinositol 3-kinase and tyrosine kinase in human eosinophils. J Leukoc Biol. 2000 Jan;67(1):117-26.

42. Kato M, Kimura H, Motegi Y, Tachibana A, Minakami H, Morikawa A, et al. Platelet-Activating Factor Activates Two Distinct Effector Pathways in Human Eosinophils. J Immunol. 2002 Nov;1(9):5252-9. 169 .

43. Nials AT, Uddin S. Mouse models of allergic asthma: Acute and chronic allergen challenge. Dis Model Mech. 2008 Nov-Dec;1(4-5):213-20.

44. Martins MA, Silva E, Castro PMR, Neto HC, Lima F, Cordeiro MCR. RSB, et al. Interactions between local inflammatory and systemic haematological effects of PAF-acether in the rat. Eur J Pharmacol. 1987 Apr 29;136(3):353-60.

45. Penagos M, Compalati E, Tarantini F, Baena-Cagnani CE, Passalacqua G, Canonica GW. Efficacy of mometasone furoate nasal spray in the treatment of allergic rhinitis. Meta-analysis of randomized, double-blind, placebo-controlled, clinical trials. Allergy. 2008 Oct;63(10):1280-91.

46. Onrust SV, Lamb HM. Mometasone furoate: A review of its intranasal use in allergic rhinitis. Drugs. 1998 Oct;56(4):725-45.

47. Yam ML, Abdul Hafid SR, Cheng HM, Nesaretnam K. Tocotrienols suppress proinflammatory markers and cyclooxygenase-2 expression in RAW264.7 macrophages. Lipids. 2009 Sep;44(9):787-97.

48. Choi Y, Lee J. Antioxidant and antiproliferative properties of a tocotrienol-rich fraction from grape seeds. Food Chem. 2009 Jun 1;114(4):1386-1390.

49. Peh HY, Ho WE, Cheng C, Chan TK, Seow ACG, Lim AYH, et al. Vitamin E Isoform Y-Tocotrienol Downregulates House Dust Mite-Induced Asthma. J Immunol. 2015 Jul;15(2):437-44. 195(.

50. Zainal Z, Abdul Rahim A, Khaza'ai H, Chang SK. Effects of Palm Oil Tocotrienol-Rich Fraction (TRF) and Carotenes in Ovalbumin (OVA)-Challenged Asthmatic Brown Norway Rats. Int J Mol Sci. 2019 Apr 10;20(7):1764.

51. Wagner JG, Harkema JR, Jiang Q, Illek B, Ames BN, Peden DB. Gamma-tocopherol attenuates ozoneinduced exacerbation of allergic rhinosinusitis in rats. Toxicol Pathol. 2009 Jun;37(4):481-91.

52. Peh HY, Tan WSD, Liao W, Wong WSF. Vitamin E therapy beyond cancer: Tocopherol versus tocotrienol. Pharmacol Ther. 2016 Jun;162:152-69.

53. Hijazi N, Abalkhail B, Seaton A. Diet and childhood asthma in a society in transition: A study in urban and rural Saudi Arabia. Thorax. 2000 Sep;55(9):775-9. 
54. Wang SY, Wang YF, Pan CC, Sun JW. Serum level and clinical significance of vitamin E in children with allergic rhinitis. BMC Pediatr. 2020 Jul;31(1):362. 20(.

55. Kongerud J, Crissman K, Hatch G, Alexis N. Ascorbic acid is decreased in induced sputum of mild asthmatics. Inhal Toxicol. 2003 Feb;15(2):101-9.

56. Ochs-Balcom HM, Grant BJB, Muti P, Sempos CT, Freudenheim JL, Browne RW, et al. Antioxidants, oxidative stress, and pulmonary function in individuals diagnosed with asthma or COPD. Eur $\mathrm{J}$ Clin Nutr. 2006 Aug;60(8):991-9.

\section{Figures}

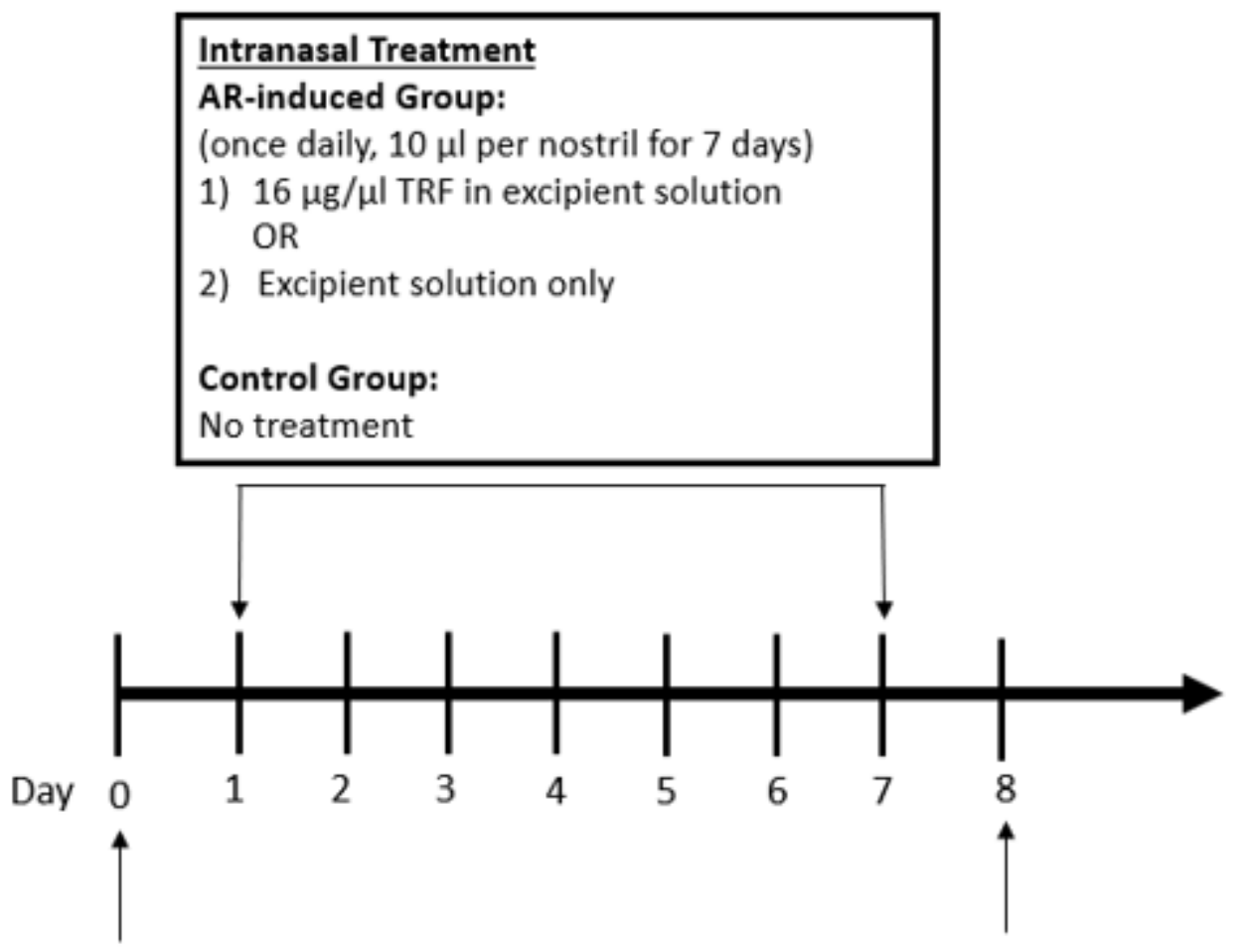

\section{AR-induced Group:}

Nasal instillation of $16 \mu \mathrm{g} / \mathrm{ml}$ PAF

at $50 \mu \mathrm{l}$ per nostril

\section{Control Group:}

Nasal instillation of vehicle at 50

$\mu$ per nostril
Anaesthetize and Sacrifice:

Collection of upper skulls

for H\&E

\section{Figure 1}

PAF-induced AR animal model. The rats were intranasally instilled with PAF on day 0 to induce AR. Treatment with TRF in excipient solution or pure excipient solution was administered intranasally to the AR rats from day 1 to 7 . No treatment was given to the control rats. All the rats were sacrificed on day 8 and upper skulls were collected for histological examination using H\&E. AR: Allergic rhinitis; H\&E: Hematoxylin and eosin; PAF: Platelet-activating factor; TRF: Tocotrienol-rich fraction. 


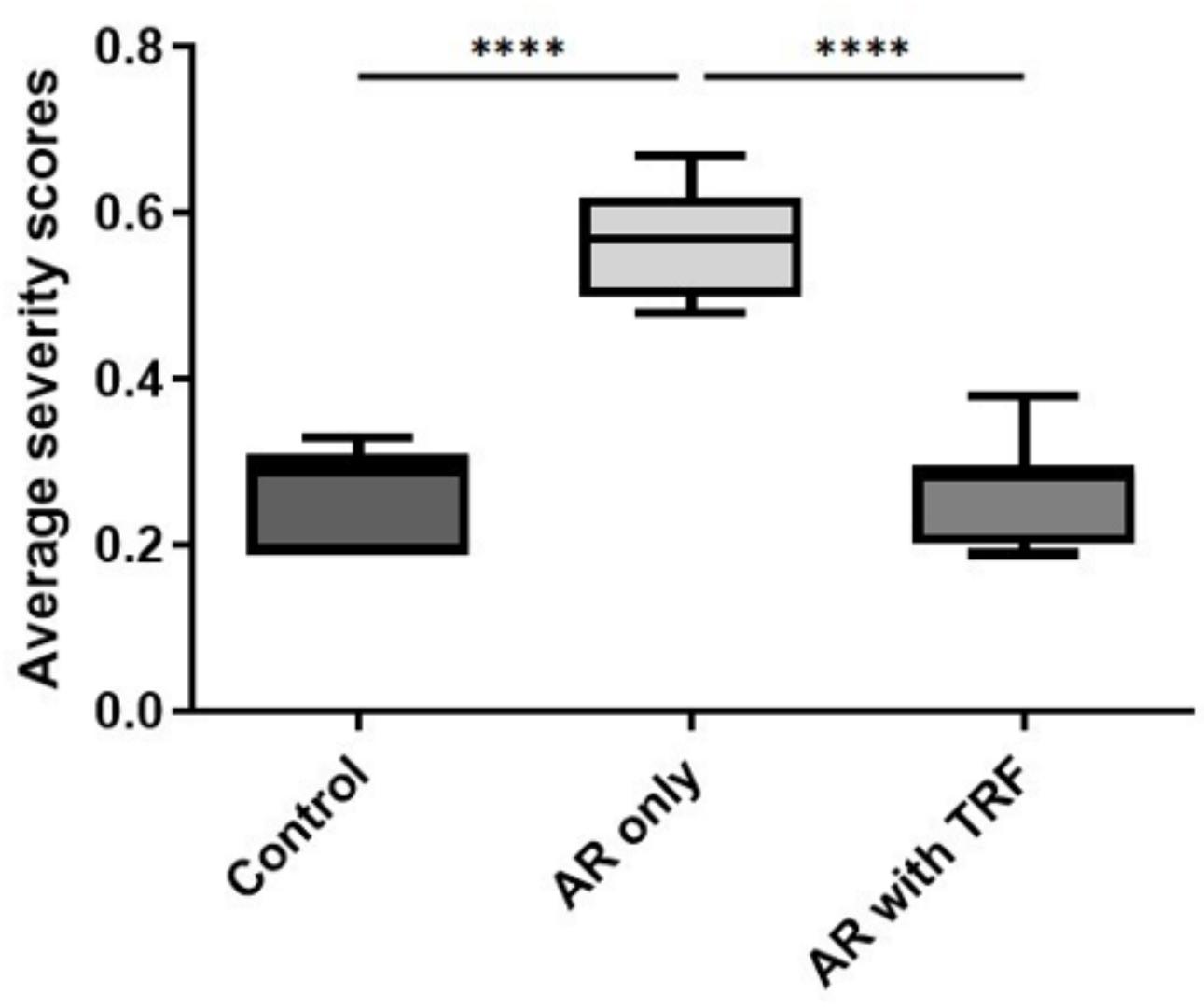

Figure 2

The average severity scores of AR in Control, PAF-induced AR and PAF-induced AR with TRF treatment groups. Data are representative of mean \pm standard error $(n=19) ; * \star \star \star p<0.0001$. AR: Allergic rhinitis; PAF: Platelet-activating factor; TRF: Tocotrienol-rich fraction.

\section{Figure 3}

The severity scores of AR for histological evaluation $(A)$ and light microscopic images of H\&E staining in nasal section (B-D; $x 200$ magnification). (B) Control group shows regular nasal structures with no inflammation. (C) PAF-induced AR group shows moderate inflammation, aggregates of polymorphonuclear cells, thickening of lamina propria and epithelial loss. (D) Regular nasal structures with minimal inflammation and aggregates of polymorphonuclear cells were observed in PAF-induced AR with TRF treatment group. Black arrows indicate inflammation and polymorphonuclear cells infiltration in the submucosa. The subjective scoring represents 0 : no significant abnormalities; 1 : minimal; 2: mild; 3 : moderate; 4: marked; 5: severe. Data are representative of mean \pm standard error $(n=19) ;{ }^{*}<<0.05$, 
$\star \star \star \star p<0.0001$. AR: Allergic rhinitis; H\&E: Hematoxylin and eosin; PAF: Platelet-activating factor; TRF: Tocotrienol-rich fraction.

A

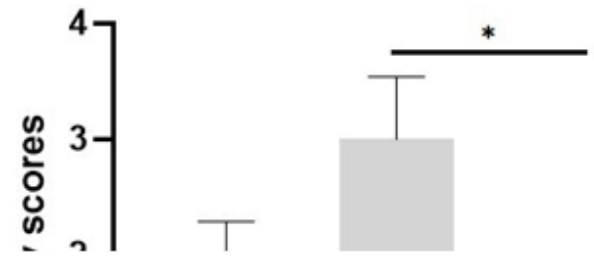

B

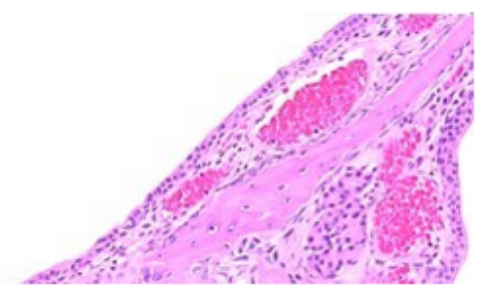

\section{Figure 4}

The severity scores of vascular congestion in submucosa $(A)$ and light microscopic images of H\&E staining in nasal section (B-D; $\times 200$ magnification). (B) Control group shows minimal to mild degree of vascular congestion. (C) PAF-induced AR groups shows moderate degree of vascular congestion. (D) PAF-induced AR with TRF treatment group shows minimal degree of vascular congestion. The subjective scoring represents 0 : no significant abnormalities; 1 : minimal; 2: mild; 3: moderate; 4: marked; 5: severe. Data are representative of mean \pm standard error $(n=19)$; ${ }^{*}<0.05$. AR: Allergic rhinitis; H\&E: Hematoxylin and eosin; PAF: Platelet-activating factor; TRF: Tocotrienol-rich fraction. 